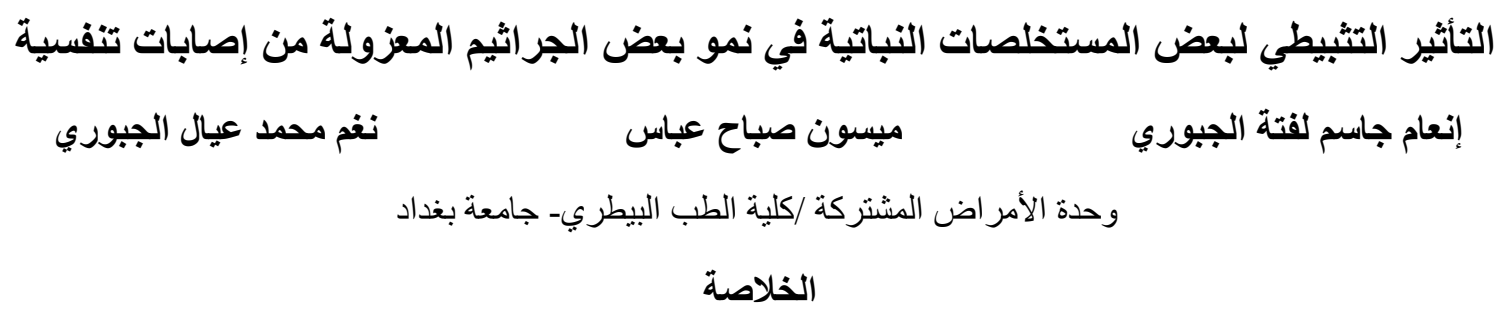

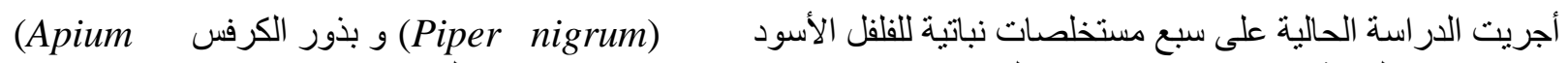

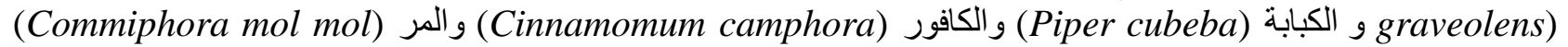

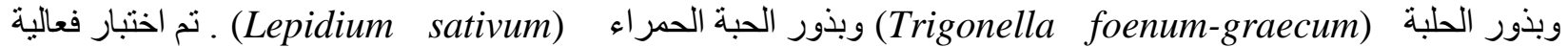

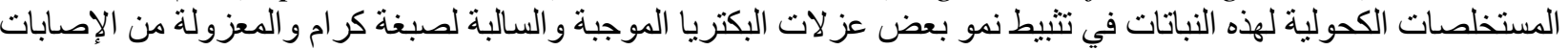

التنفسية وهي: Streptococcus pneumoniae و و Micrococcus antarcticus و و Coagulase- negative و Neisseria meningitides و و المكورات السحائية و K. pneumoniae و Klebsiella oxytoca و Pseudomonas aeruginosa و Staphylococci (CNS) كنلك جرى دراسة حساسية البكتريا لـ 10 أنواع من المضادات الحيوية هي: . Escherichia coli Cloxacillin و Ofloxacin (OFX) Ampicillin (AM) و Vancomycin (VA) , Ceftazidime (CAZ) Amoxicillin/Clavulanic, Oxytetracycline (E) , Erythromycin (E) , Cefalothin (KF) , (CX) كرام. acid(CTX)

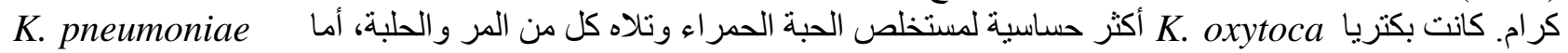

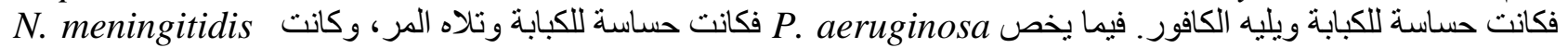

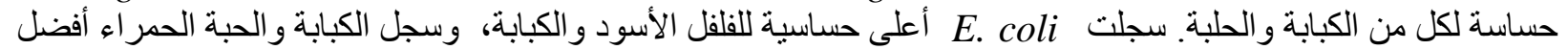
النتائج ضد aureus كنللك الحال في antarcticus

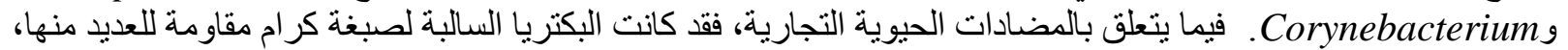

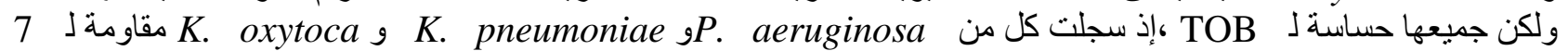

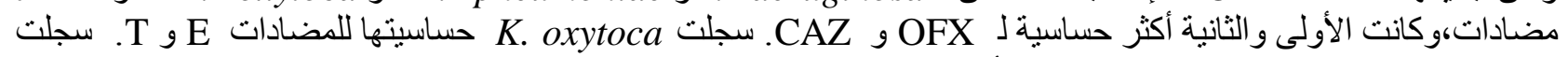

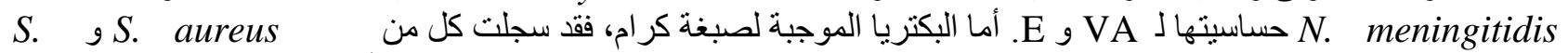

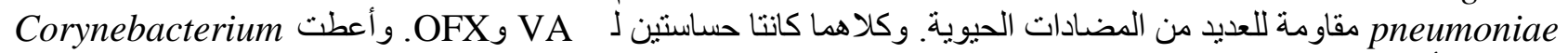
حساسية لأربعة مضادات هي: TOB و VA و V و

\title{
The inhibitory effect of some medical plants on the growth of some bacterial isolates from respiratory infections
}

\author{
Al-Jebouri, A.J \\ Abbas, M.S. \\ Eaal, N.M.
}

Accepted -October - 2010

\section{Summary}

This study was carried out to evaluate seven types of herbs being: black pepper seeds (Piper nigrum), celery seeds (Apium graveolens), cubeb seeds (Piper cubeba), camphor seeds (Cinnamomuum camphora), myrrh (Commiphora mol mol), fenugreek seeds (Trigonella foenumgraecum) and seeds of Lepidium sativum. The antibacterial activity of alcoholic extracts of these plants was performed on gram- positive and gram- negative bacteria isolated from human respiratory tract infections including : $S$. aureus , S. pneumoniae, $M$. antarcticus, Corynebacterium spp. and coagulase- negative Staphylococci (CNS). Gram -negative bacteria included: K. oxytoca, $K$. pneumoniae and $N$. meningitidis, $P$. aeruginosa and $E$. coli. We studied the bacterial sensitivity to 10 antibiotics: Tobramycin (TOB), Ceftazidime (CAZ), Vancomycin (VA), Ampicillin (AM), Ofloxacin (OFX), Cloxacillin (CX), Cefalothin (KF), Erythromycin (E), Oxytetracycline (T), Amoxicillin/Clavulanic acid (CTX). Cubeba essential oil was superior in inhibiting all the examined bacteria compared with other plants. $K$. oxytoca was most sensitive to Lepidium sativum seeds extract followed by myrrh and fenugreek, while $K$. pneumoniae was most sensitive to cubeba 
and camphora. Cubeba followed by myrrh pi spp. was resistant to 5 antibiotics and sensitive to 4 which were: TOB, VA, OFX and KF.

Key Words: medical plant, respiratory infections piper cubeba, apium graneolens.,

\section{المقدمة}

تسبب جر اثيم المكور ات العنقودية الذهبية S. aureus العديد من الإصابات القيحية سواء بالإنسان أو الحيوان، فهي توجد بمثابة فلور ا طبيعية بالقناة التنفسية العليا، كذلك الحال في

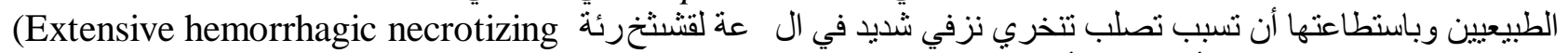
فض consolidation) في جر اثيم E. coli و الزو ائف الزنجارية P. aeruginosa فيتنتر بصورة واسعة في البيئة وتسبب ذات الرئة المكتسب

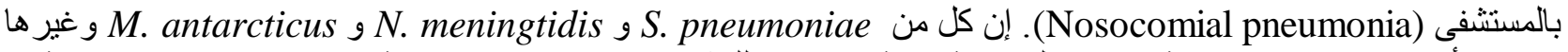

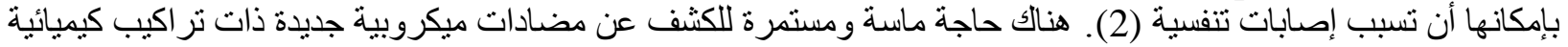

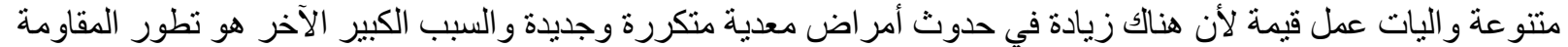

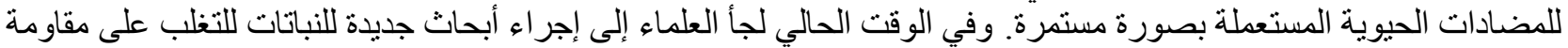

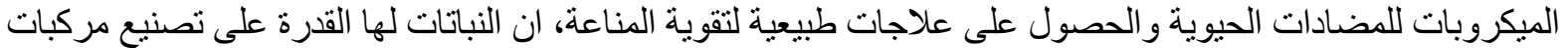

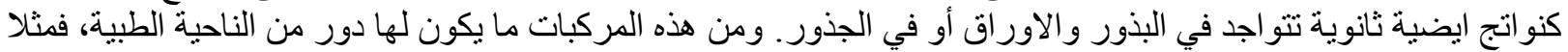

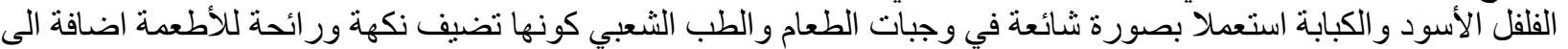

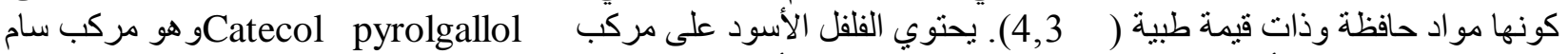

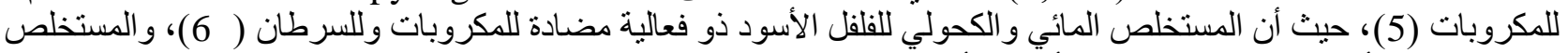

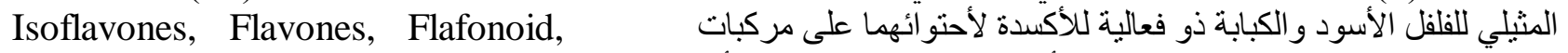
Coumarin anthocycinin

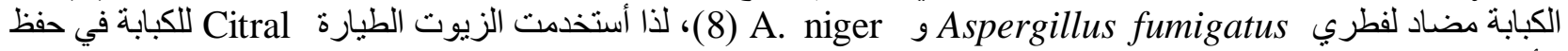

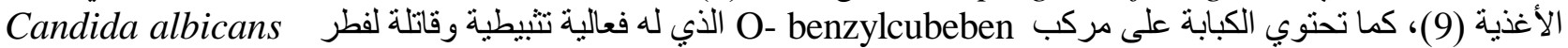

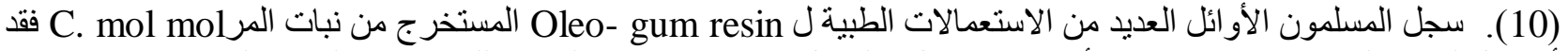

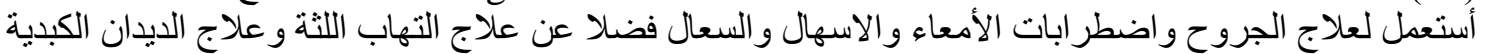

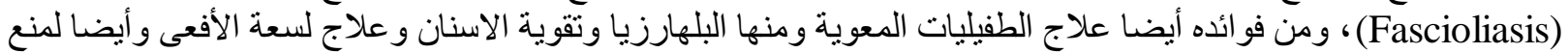

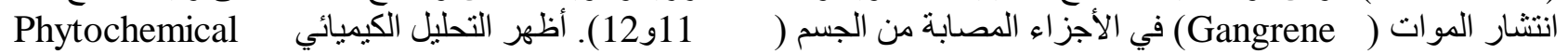

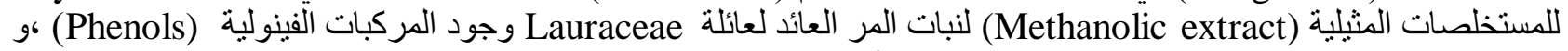

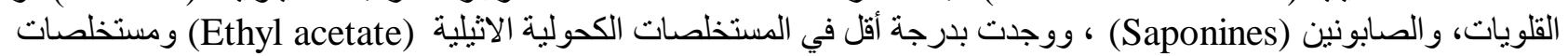

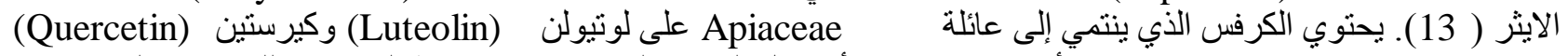

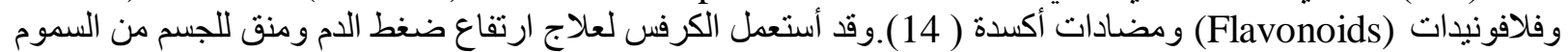

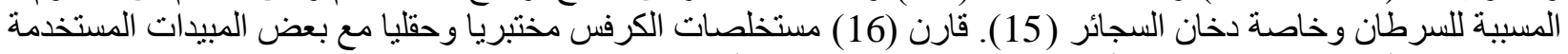

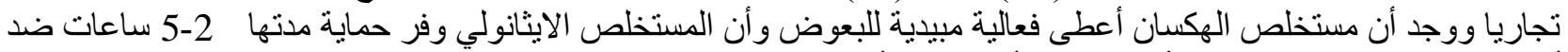

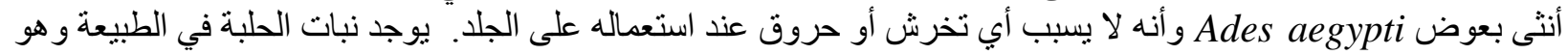

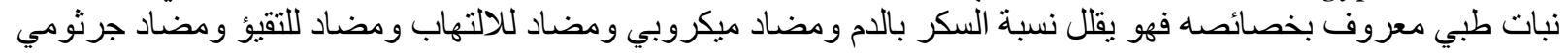

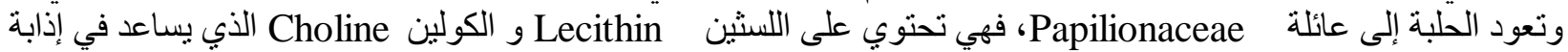

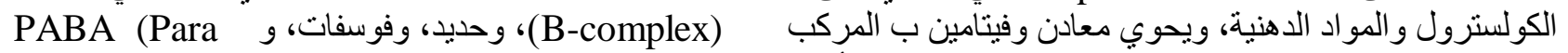

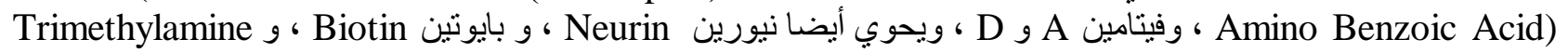

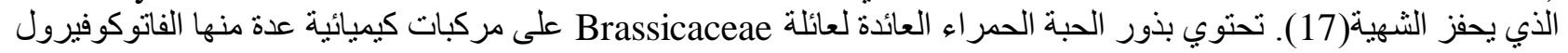

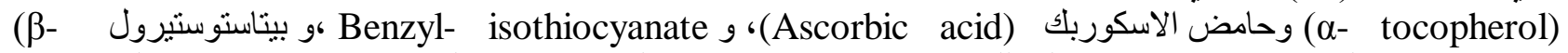

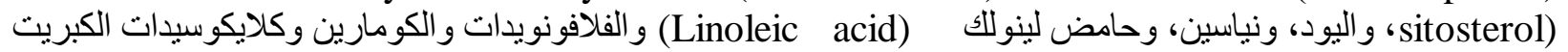

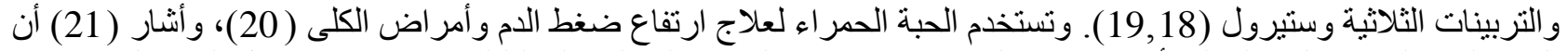

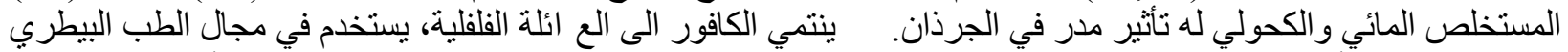

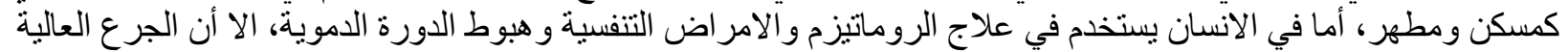

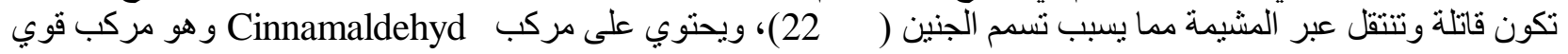




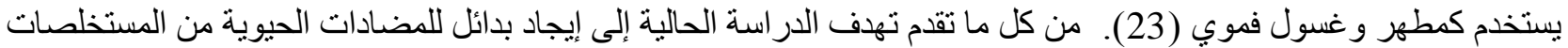

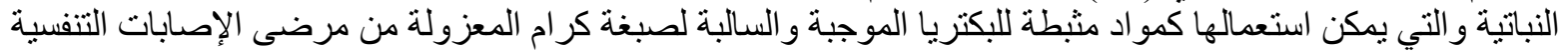

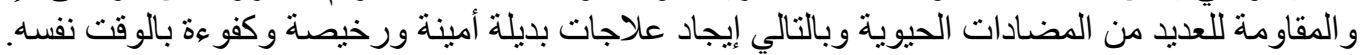

\section{المواد وطرائق العمل}

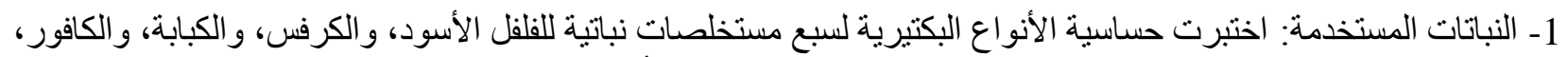

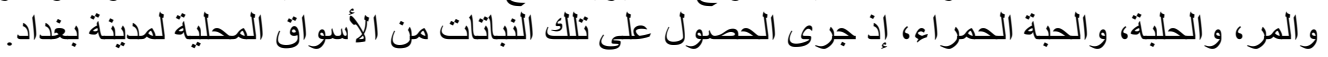

2- تحضير المستخلصات النباتية: حضرت المستخلصات النباتية حسب طريقة ( 24) كآلاتي: جرى طدن البذور كل على حدة

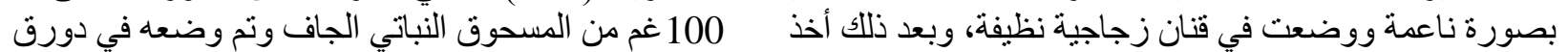

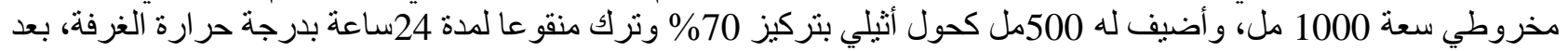

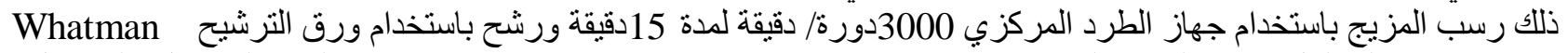

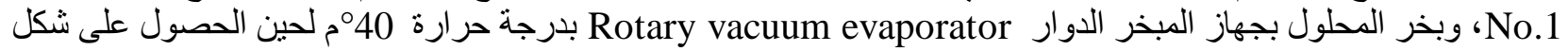

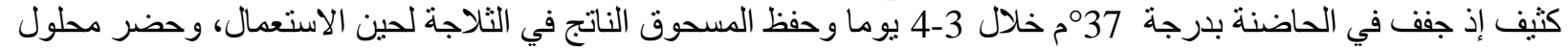

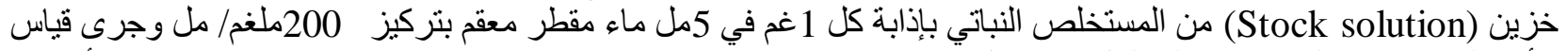

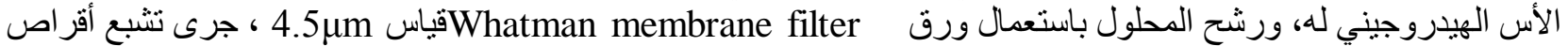
من ورق الترشيح بالمستخلص الكحولي للنباتات المختلفة.

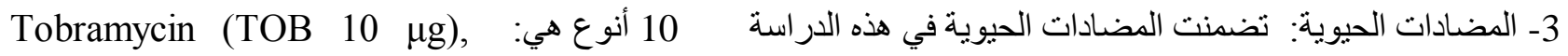
Ceftazidime (CAZ $30 \mu \mathrm{g}$ ), Vancomycin (VA $30 \mu \mathrm{g}$ ), Ampicillin (AM $10 \mathrm{mcg} / \mathrm{disc}$ ), Ofloxacin (OFX $5 \mu \mathrm{g}$ ), Cloxacillin (CX $1 \mu \mathrm{g}$ ), Cefalothin (KF $30 \mu \mathrm{g}$ ), Erythromycin (E $15 \mu \mathrm{g}$ ), Oxytetracycline (T $30 \mu \mathrm{g})$, Amoxicillin/ clavulanic acid (CTX $30 \mu \mathrm{g})$.

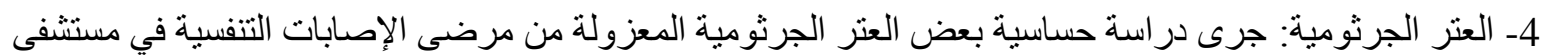

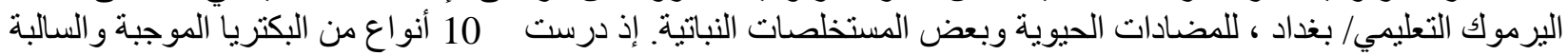

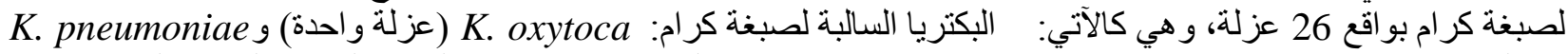

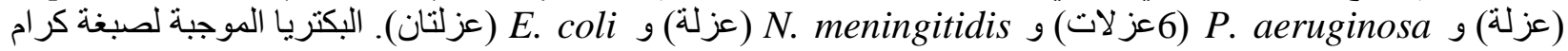

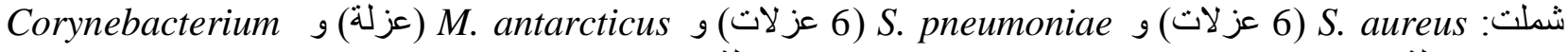
spp.

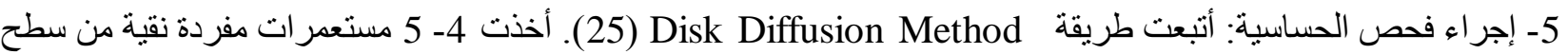

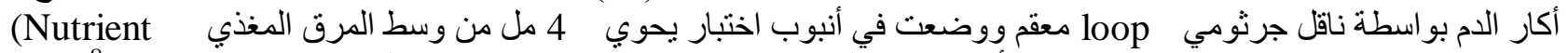

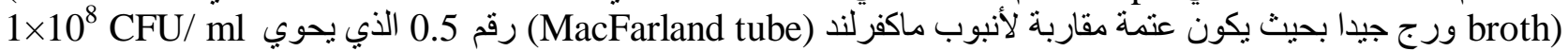

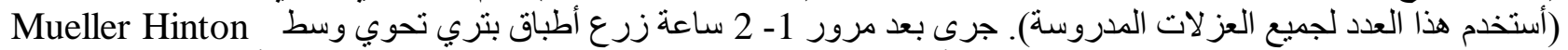

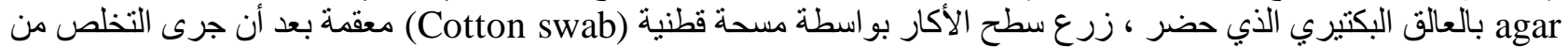

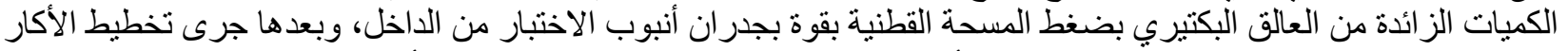

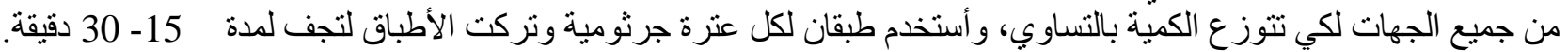

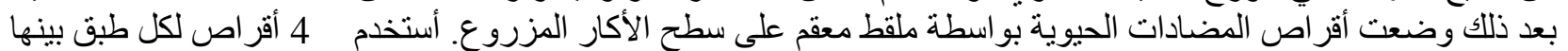

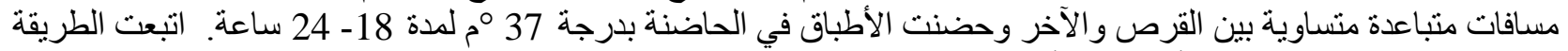

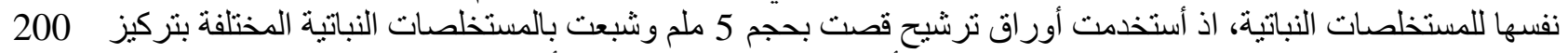
مايكرو غر ام/ مل. جرى قياس قطر منطقة التثبيط حول أقر اص المضادات الحيوية و أقراص المستخلصات النباتية بالمليمنر بو اسطة المسطرة الاعتيادية. 


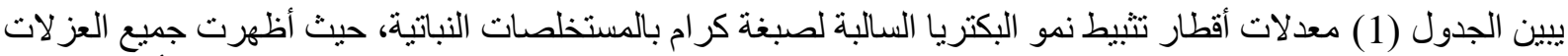

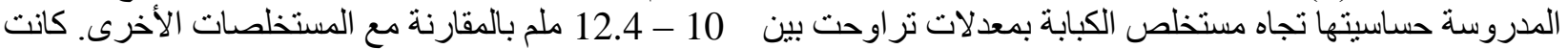
الحبة السوداء والحلبة والمر الأفضل بين المستخلصات الأخرى في تثبيط نمو 10 oxytoca بأقطار تثيط التيط 20 و 12 و 12 على الى

الجدول (1): معدلات أقطار تثبيط المستخلصات النباتية بالملم تجاه البكتريا السالبة لصبغة كرام

\begin{tabular}{|c|c|c|c|c|c|c|c|}
\hline \multicolumn{7}{|c|}{ معدلات أقطار تثبيط المستخلصات (بالملم) } & \\
\hline الحبة الحمراء & الحلبة & المر - المر & الكافور & الكبابة & الكرفس & الفلفل الأسود & البكتريا \\
\hline 20 & 12 & 12 & 6 & 10 & 5 & 5 & K. oxytoca \\
\hline 5 & 5 & 5 & 9 & 11 & 5 & 5 & K. pneumonia \\
\hline 7.9 & 6.7 & 8.3 & 7.4 & 12.4 & 5.7 & 5.9 & P. aeruginosa \\
\hline 5 & 10 & 5 & 5 & 10 & 6 & 5 & N. meningitides \\
\hline 5 & 5 & 5 & 5.5 & 10 & 5.5 & 10 & E. coli \\
\hline
\end{tabular}

كانت جميع العز لات البكتيرية الموجبة لصبغة كر ام حساسة لمستخلص الكبابة، الذي سجل معدلات بلغت 12 ملم في كل من

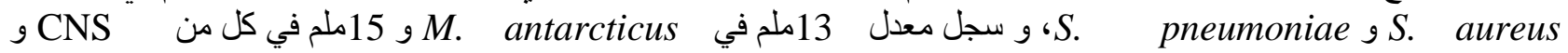
Corynebacterium ، كما موضح في (الجدول 2). الجدول (2): معدلات أقطار تثبيط المستخلصات النباتية بالملم تجاه البكتريا الموجبة لصبغة كرام

\begin{tabular}{|c|c|c|c|c|c|c|c|}
\hline \multicolumn{7}{|c|}{ معدلات أقطار تثبيط المستخلصات (بالملم) } & \\
\hline الحبة الحمراء & الحلبة & 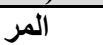 & الكافور & الكبابة & 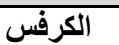 & الفلفل الأسود & البكتريا \\
\hline 12.5 & 10 & 8.75 & 11.25 & 12 & 5.5 & 6 & S. aureus \\
\hline 6.16 & 7 & 6.16 & 8.5 & 12 & 6.66 & 5.5 & S.pneumoniae \\
\hline 5 & 5 & 5 & 6 & 15 & 5 & 5 & CNS \\
\hline 15 & 6 & 6 & 10 & 13 & $\mathbf{1 0}$ & 5 & M. antarcticus \\
\hline 5 & 5 & 5 & 8 & 15 & 5 & 5 & Corynebacterium \\
\hline
\end{tabular}

يبين الجدول (3) معدلات أقطار تتبيط 10 من المضادات الحيوية لنمو خمسة أنو اع بكتبرية، اذ كان المضاد الحيوي TOB فئاء

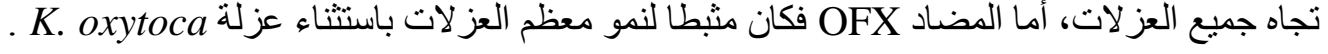
الجدول (3): معدلات أقطار تثبيط المضادات الحيوية بالملم تجاه البكتريا السالبة لصبغة كرام

\begin{tabular}{|c|c|c|c|c|c|}
\hline & \multicolumn{5}{|c|}{ Mean Zones of Inhibition of Antibiotics (mm) } \\
\hline Antibiotic & K. oxytoca & K. pneumoniae & P. aeruginosa & N.meningitidis & E. coli \\
\hline TOB & $15 \mathrm{~S}$ & $25 \mathrm{~S}$ & $21.5 \mathrm{~S}$ & $40 \mathrm{~S}$ & $18 \mathrm{~S}$ \\
\hline CAZ & $-\mathbf{R}$ & $24 \mathrm{~S}$ & $22.6 \mathrm{~S}$ & $-\mathbf{R}$ & $19 \mathrm{R}$ \\
\hline VA & $-\mathbf{R}$ & $-\mathbf{R}$ & $-\mathbf{R}$ & $23 \mathrm{~S}$ & $-\mathbf{R}$ \\
\hline AM & $-\mathbf{R}$ & $-\mathbf{R}$ & $-\mathbf{R}$ & $-\mathbf{R}$ & $-\mathbf{R}$ \\
\hline OFX & $8 \mathbf{R}$ & $30 \mathrm{~S}$ & $23 \mathrm{~S}$ & $19 \mathrm{~S}$ & $23.5 \mathrm{~S}$ \\
\hline $\mathbf{C X}$ & $-\mathbf{R}$ & $-\mathbf{R}$ & $-\mathbf{R}$ & $-\mathbf{R}$ & $-\mathbf{R}$ \\
\hline KF & $-\mathbf{R}$ & $-\mathbf{R}$ & $-\mathbf{R}$ & $-\mathbf{R}$ & $-\mathbf{R}$ \\
\hline $\mathbf{E}$ & $25 \mathrm{~S}$ & $-\mathbf{R}$ & $-\mathbf{R}$ & $25 \mathrm{~S}$ & $-\mathbf{R}$ \\
\hline $\mathbf{T}$ & $19 \mathrm{~S}$ & $11 \mathrm{R}$ & $-\mathbf{R}$ & $11 \mathrm{R}$ & $16.5 \mathrm{Int}$ \\
\hline AMC & $-\mathbf{R}$ & $-\mathbf{R}$ & $-\mathbf{R}$ & 14 Mod. S. & $-\mathbf{R}$ \\
\hline
\end{tabular}

R: resistant; S: sensitive; Int.: intermediate; Mod. S: moderately susceptible. 


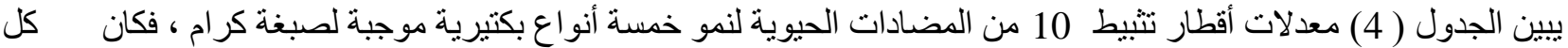

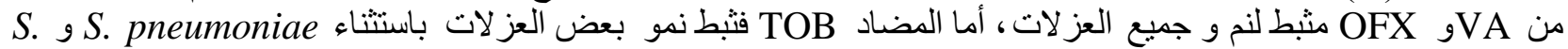
aureus

جدول (4) : معدلات أقطار تثيط المضادات الحيوية بالملم تجاه البكتريا الموجبة لصبغة كرام

\begin{tabular}{|c|c|c|c|c|c|}
\hline & \multicolumn{5}{|c|}{ Mean Zones of Inhibition of Antibiotics (mm) } \\
\hline Antibiotic & S. aureus & S. pneumoniae & CNS & M. antarcticus & Corynebacterium \\
\hline TOB & $10.6 \mathrm{R}$ & $7.6 \mathrm{R}$ & $17 \mathrm{~S}$ & $16 \mathrm{~S}$ & $16 \mathrm{~S}$ \\
\hline CAZ & $\mathbf{R}$ & $\mathbf{R}$ & $\mathbf{R}$ & $23 \mathrm{~S}$ & $-\mathbf{R}$ \\
\hline VA & $17.3 \mathrm{~S}$ & $19.16 \mathrm{~S}$ & $33 \mathrm{~S}$ & $28 \mathrm{~S}$ & $17 \mathrm{~S}$ \\
\hline $\mathbf{A M}$ & $8.3 \quad \mathrm{R}$ & $18.16 \quad R$ & $22 \mathbf{R}$ & $40 \mathrm{~S}$ & $20 \mathbf{R}$ \\
\hline OFX & $18.16 \mathrm{~S}$ & $18.8 \mathrm{~S}$ & $24 S$ & $31 \mathrm{~S}$ & $18 \mathrm{~S}$ \\
\hline $\mathbf{C X}$ & $\mathbf{9} \mathbf{R}$ & $\mathbf{R}$ & $15 \mathrm{~S}$ & 27 S & $-\mathbf{R}$ \\
\hline KF & $6.6 \mathrm{R}$ & $9.8 \quad R$ & $-\mathbf{R}$ & $40 \mathrm{~S}$ & $21 \mathrm{~S}$ \\
\hline $\mathbf{E}$ & $9.4 \mathrm{R}$ & 18.2 Int. & $\mathbf{N}$ & $\mathbf{N}$ & 18 Int. \\
\hline $\mathbf{T}$ & 15.75 Int. & $9.8 \quad R$ & $\mathbf{N}$ & $\mathbf{N}$ & $10 \mathbf{R}$ \\
\hline AMC & $-\mathbf{R}$ & $\mathbf{R}$ & $\mathbf{N}$ & $\mathbf{N}$ & $9 R$ \\
\hline
\end{tabular}

N: not used; R: resistant; S: sensitive; CNS: coagulase- negative Staphylococci; Int.: intermediate. المناقثة

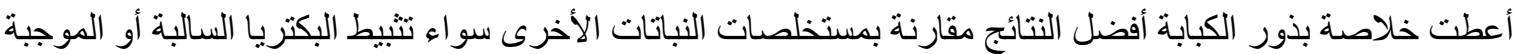

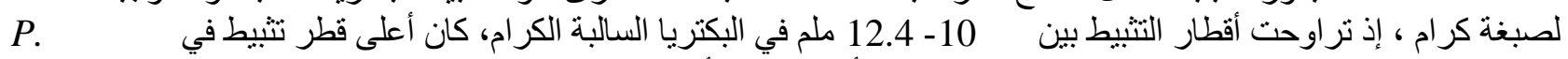
aeruginosa

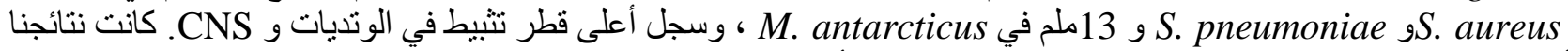

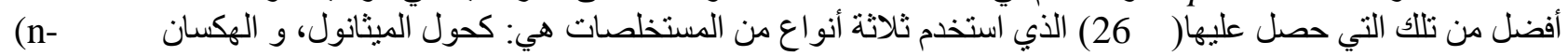

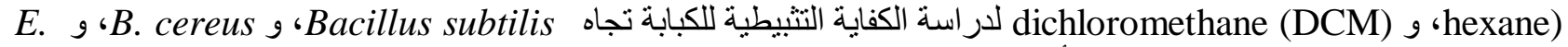

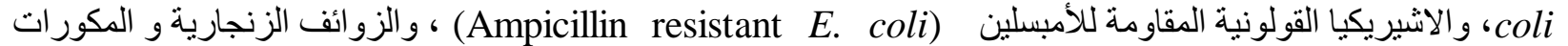

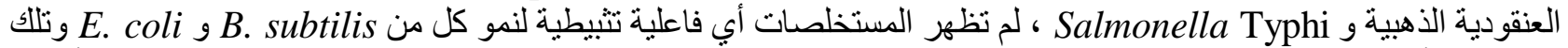

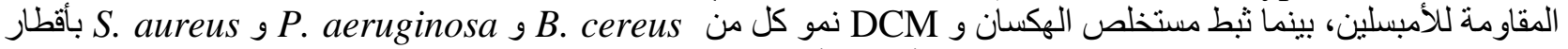
تثبيط 12 و 10 و 17 ملم على التو الي للمستخلص الأول ، و أقطار تثيط 23 و و 8 و و 16 ملم بالترتيب للمستخلص الثناني. بينما

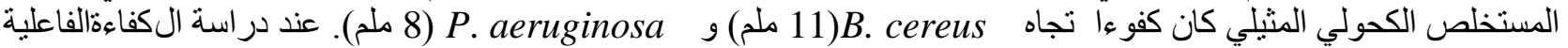

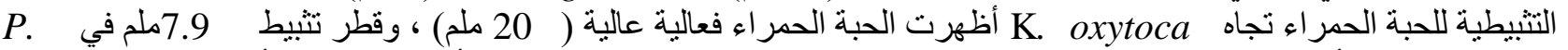
والكول aeruginosa و الكحولي المثيلي لبذور الحبة الحمر اءلم تظهر أي نتيجة تذكر عند اختبار فاعليتها تجاه

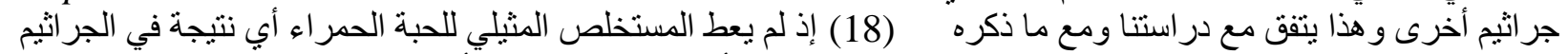

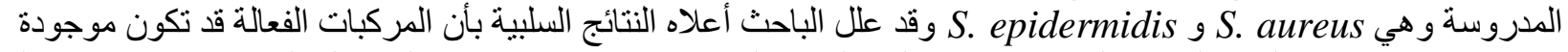

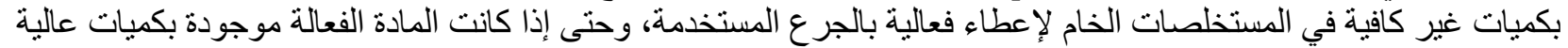

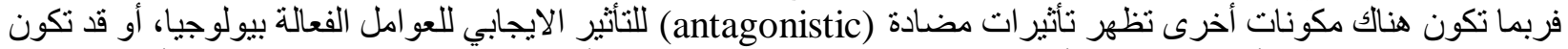

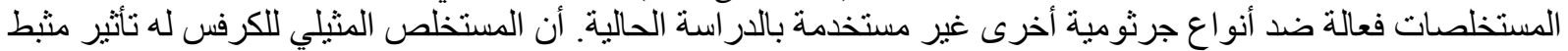

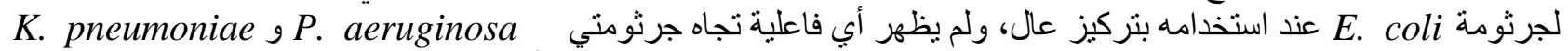

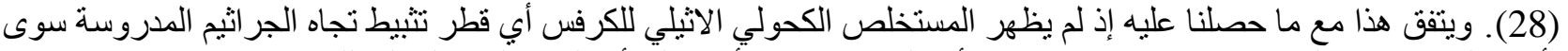

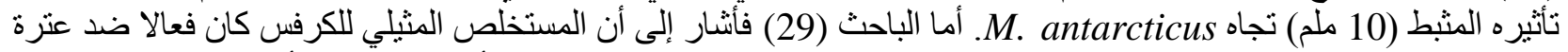

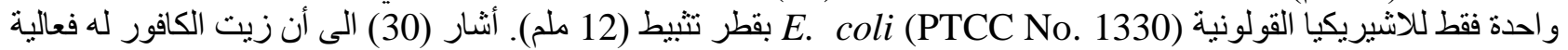

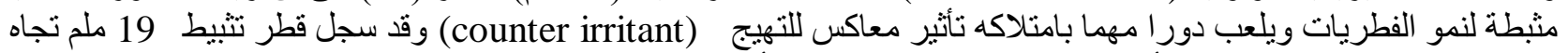

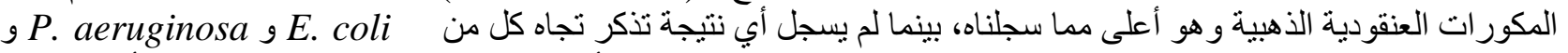

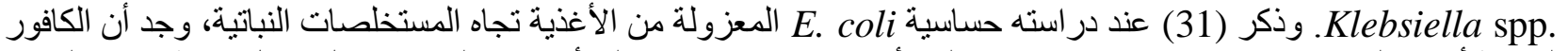

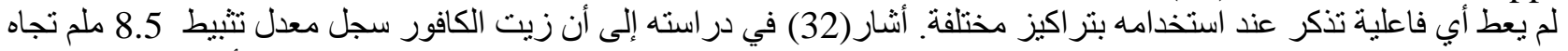

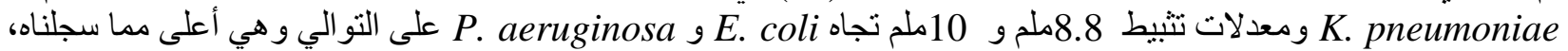
في حين لم يسجل أي قطر تنبيط تجاه S. aureus على النقيض من در استنا. 
استخدمت التربينات المستخلصة من نبات المر كمثبط لنمو S. aureus (33). و وجد حديثا أن المر يمتلك فعاليات مضادة تجاه

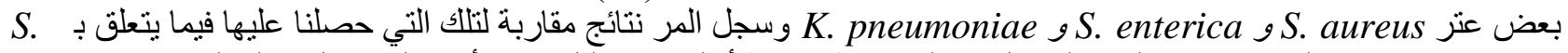
aureus

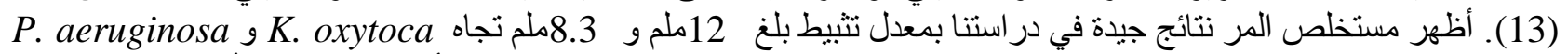

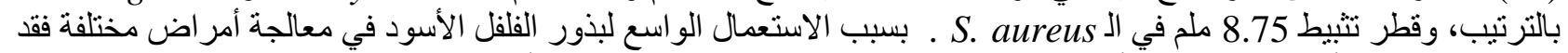

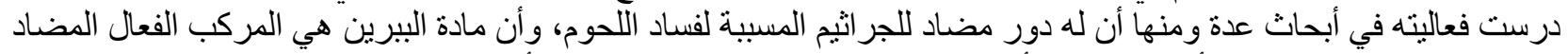

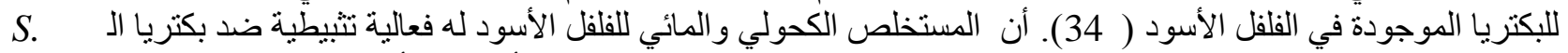
S. S. S المقاومة للبنسلين Gureus P. و و و aureus الج aeruginosa

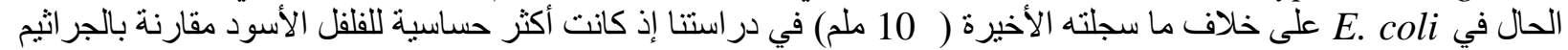

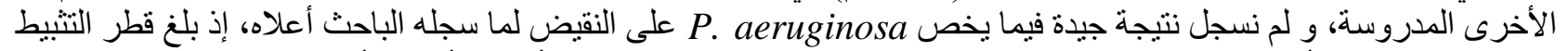

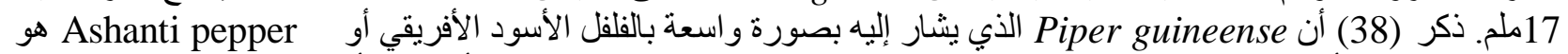



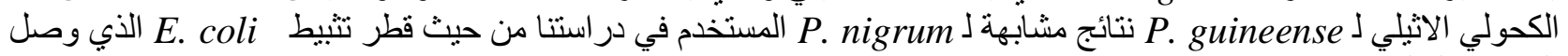
إلى 10 ملم لكلاهما.

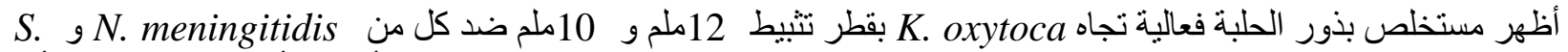



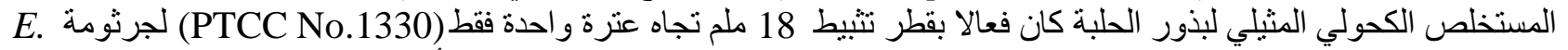

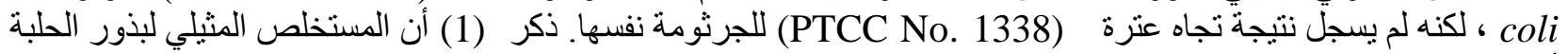

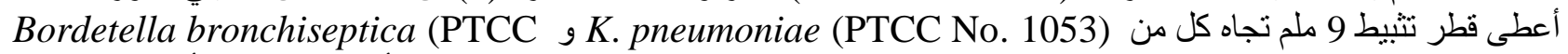

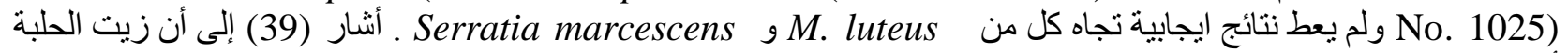
أظهر تثبيطا قويا تجاه

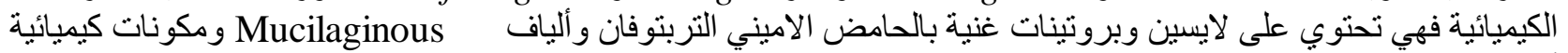

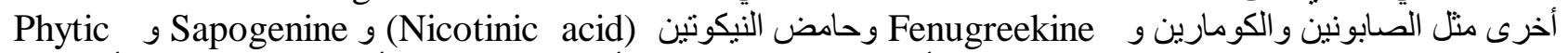
و Scopoletin acid خصائص مضادة للبكتريا من خلال إعاقة قوة حركة البرونون Proton Motive Force (PMF) مسببة بذللك تسرب الإبة المكونات

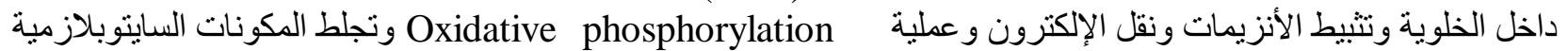

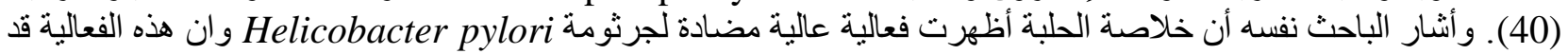

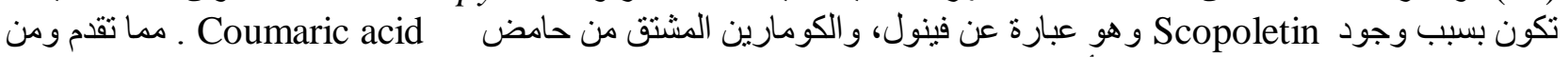

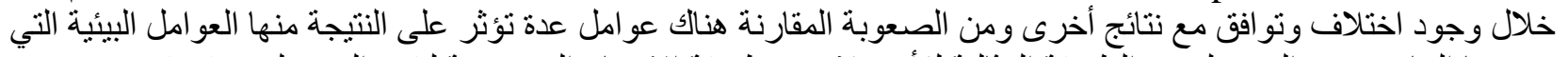
نمى بها النبات ونوع المستخلص و الطريقة المثالية للأستخلاص وطريقة الكتة الاختبار المستخدمة لتقييم المستخلص (41).

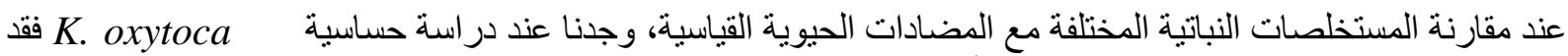

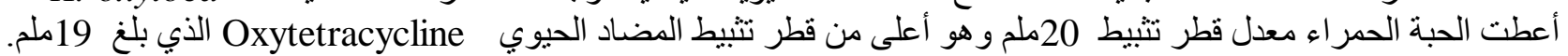

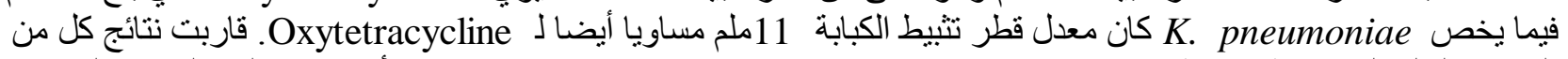

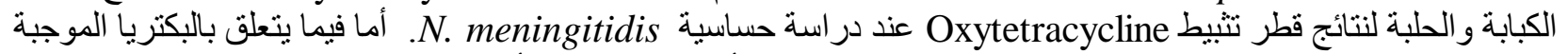

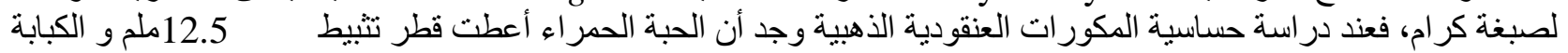

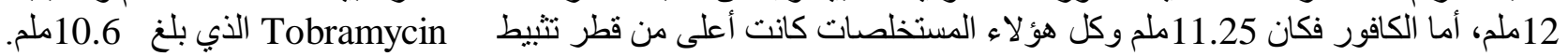

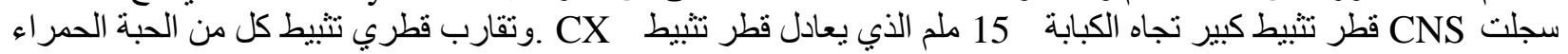

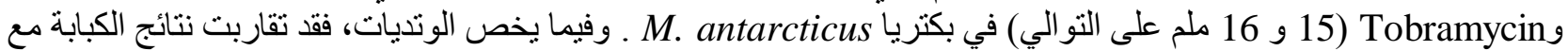

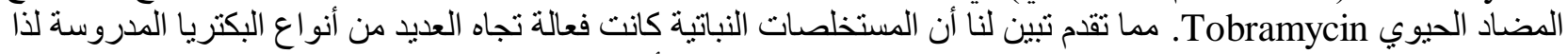

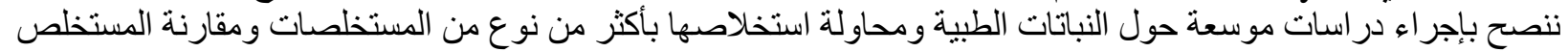
المائي و الكحولي الاثيلي و المثيلي و غير ها من المستخلصات لكل منها لغرض استعمالها كعلاجات بديلة للمضادات التهات الحيوية. 


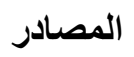

1- Bonjar GHS (2004a). Evaluation of antibacterial properties of Iranian medicinal plants against Micrococcus luteus, Serratia marcescens, Klebsiella pneumoniae and Bordetella bronchiseptica . Asian J Plant Sciences, 3 (1): 82- 86.

2- Winn WC Allen SD Janda WM Koneman EW Procop GW Schreckenberger PC and Woods GL (2006). Koneman 's Color Atlas and Textbook of Diagnostic Microbiology. $6^{\text {th }}$ ed. Lippincott William 's and Wilkins Company, Philadelphia.

3- DeSouza EL Stamford TL and Lima EO (2005). Antimicrobial effectiveness of spices: an approach for use in food conservation system. Braz Arch Biol Technol. 48(4): 1516-8913.

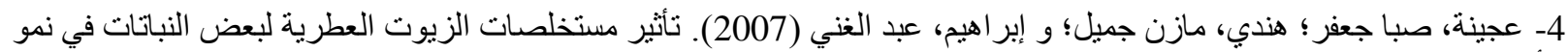

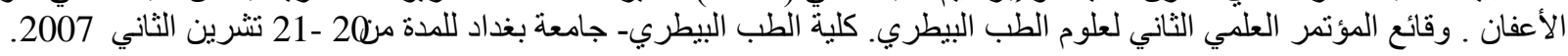

5- Cowan MM (1999). Plant products as Antimicrobial Agents. Clin. Microbiol. Rev. 564-582.

6- Aqil F Ahmed I Mehmood Z (2006). Antioxidant \& free radical scavenging properties of twelve traditionally used Indian medicinal plants. Turk J Biol. 30: 177-183.

7- Khalaf NA Shakya A K AL- Othman EL- Agbar Z. and Farah H (2008). Antioxidant activity of some commen plants. Turk J Biol. 32: 51-55.

8- Balacs T(1991). Research reports (Fungal inhibition). Int J Aromatherapy. 3:30.

9- Balacs T(1992). Research reports (Fungal inhibition). Int J Aromatherapy. 4:31.

10- Silva ML Coimbra HS Pereira ALima TC (2007). Evaluation of piper cubeba extract, (-)-cubebin \& its semi-synthetic derivatives against oral pathogens. PTR. Phtotherapy research. 21: 420-422.

11- Khan A (2006). Aloe and Myrrh: modern day analysis of two ancient herbs. Tariq Magazine. 11(2): 36-37.

12- Sarah M (2004). Myrrh: magi, medicine \& mortality. Pharmaceutical. J. 273: 919-921.

13- Abdallah EM Khalid AS and Ibrahim N (2009). Antibacterial activity of oleo - gum resins of Commiphora molmol and Boswellia papyrifera against methicillin resistant Staphylococcus aureus (MRSA). Scientific Research and Essays. 4(4): 351- 356.

14- Kolarovic J Popovic M Mikov M Mitic R and Gvozdenovic L (2009). Protactive effects of celery juice in treatments with Doxorubicin. Molecules, 14: 1627-1638.

$$
\text { 15- السرميطي، رشا (2008). صيدلية الأعشاب. شبكة النظم العربية. }
$$

16- Tuetun B Choochote W Kanjanapothi D and Pitasawat B (2005). Repellent properties of celery, Apium graveolensl, compared with commercial repellents, against mosquitoes under laboratory \& field conditions. Trop. Med. \& Int. Health. 10(11): 1190-1198.

17- Khan FU Durrani FR Sultan A Khan RU and Naz S (2009). Effect of Fenugreek (Trigonella foenum- graecum) seed extract on visceral organs of broiler chicks. ARPN. J. Agricult Biolo Sci. 4, No.1.

18- Parekh J and Chanda S (2008). Antibacterial activity of aqueous and alcoholic extracts of 34 Indian medicinal plants against some Staphylococcus spp. Turk J Biol. 32: 63-71. 
19- Radwan HM El -Missiry MM Al- Said WM and Ismail AS (2007). Investigation of the glucosinolates of Lepidium sativum growing in Egypt and their biological activity. Res Med Sci. 2(2): 127- 132 .

20- Jouad H Haloui M and Elhilaly J(2001). Ethobotanical Survy of medicinal plants used for the treatment of diabetes, cariac and renal diseases in the North center region of Morocco (EeZBoulemane). J. Ethopharmacol. 77: 175- 182.

21- Patel U Kulkarni M Undal V and Bhosale A (2009). Evaluation of Diuretic activity of aqueous and methanol extracts of Lepidium sativum garden cress (Cruciferae) in Rats Trop. J Pharmaceutical Res. 8: 215- 219.

22- Committee For Veterinary Medicinal Products, Camphora (use in veterinary homeopathy). 1999.

23- Wallace JR (2004). Antimicrobial properties of plant secondary metabolites. Pro. Nutr. Soc. 63: 621- 629 .

24- Anessiny G and Perez C (1993). Screening of plants used a green line. Folk medicine for antimicrobial activity. J Ethnopharmacol. 39: 119-128.

25- Harley JP and Prescott LM (2002). Laboratory Exercises in Microbiology. $5^{\text {th }}$ ed. The Mc GrowHill Companies. USA.

26- Chitnis R Abichandani M Nigam P Nahar L and Sarker SD (2007). Antioxidant and antibacterial activity of the extracts of Piper cubeba (Piperaceae). Ars. Pharm. 48 (4): 343 - 350.

27-Parekh J. and Chanda S (2007). In vitro screening of antibacterial activity of aqueous and alcoholic extracts of various Indian plant species against selected pathogens from Enterobacteriaceae. African $\mathbf{J}$ Microbiol. Res.1 (6): 92- 99.

28- Akroum S Satta D and Lalaoui, K. (2009). Antimicrobial, antioxidant, Cytotoxic activities and phytochemical screening of some Algerian plants. Europ J Sci Res. 32 (2): 289 - 295.

29- Bonjar GHS (2004 b). Screening for antibacterial properties of some Indian plants against two strains of Escherichia coli. Asian J Sci. 3(3): 310 - 314.

30- Vidya, T. J. and Vidya, P. (2000). Antimicrobial activity of Scavon Vet. Cream. The Veterinarian. (2000): (24), August, 16.

31- Lee J Lee J Lim J Sim S and Park D (2008). Antibacterial effects of S-(-)-tulipalin B isolated from Spiraea thunbergii Sieb on Escherichia coli, a major food borne pathogenic microorganism. J. Med plants Res .2 (3): 59- 65.

32- Prabuseenivasan S Jayakumar M and Ignacimuthu S (2006). In vitro antibacterial activity of some plant essential oils. BMC Comlementary and alternative medicine. 6: 39.

33- Rahman MM Garvey M and Gibson S (2008).Antibacterial terpenes from the Oleo-resin of Commiphora molmol.Phytotherapy Research. Aug.11.

34- Outara B Simard RE and Holley RA (1997). Antibacterial activity of selected fatty acids and essential oils against six meat spoilge organisms. Int $\mathbf{J}$ food. Microb. 37: 155- 162.

35- Perez C and Anesini C (1994). Antibacterial activity of alimentary plants against Staphylococcus aureus growth. Am. J Chin. Med. 22: 169- 174.

36- Masood N Chaudhry A and Tariq P (2006). Bactericidal activity of Black pepper, Bay leaf, Aniseed and Coriander against oral isolates. Pak J Pharm Sci. 19(3): 214- 218. 


\section{المجلة الطبية البيطرية العراقية 34 ( 2 ): 117 - 125 ، ( 2010 ).}

37- Abdel Gadir WS Mohamed F and Bakhiet MO (2007). Antibacterial activity of Tamarindus indica fruit and Piper nigrum seeds. Res J Microbiol. 2(11): 824- 830.

38- Nwinyi OC Chinedu NS Ajani OO Ikpo CO and Ogunniran KO (2009). Antibacterial effects of extracts of Ocium gratissimum and Piper guineese on Escherichia coli and Staphylococcus aureus. African J Food Sci. 3(3): 77- 81.

39- Wagh P Rai M Deshmukh SK and Durate MCT (2007) .Bio- activity of oils of Trigonella foenum - graecum and Pongamia pinnata. African J Biotech. 6(13): 1592- 1596.

40- Randhir R Lin YT and Shetty K (2004). Phenolics, their antioxidant and antibacterial activity in dark germinated fenugreek sprouts in response to peptide and phytochemical elicitors .Asia Pac J Clin Nutr. 13 (3): 295- 307.

41- Nostro A Germano MP and Marino A (2000). Plant antimicrobial activity. Lett. Microbiol. 30(1): 379- 384 . 